\section{Kniearthrose bei Patienten mit hoher Knochendichte}

\author{
Hartley A et al. Individuals with high bone mass \\ have increased progression of radiographic and \\ clinical features of knee osteoarthritis. \\ Osteoarthritis Cartilage 2020; 28: 1180-1190
}

Bei Kniegelenksarthrose treten häufig Osteophyten und Gelenkspaltverschmälerung auf. Außerdem besteht ein Zusammenspiel mit der Knochendichte. Studien weisen auf eine inverse Beziehung zwischen Osteoporose und Arthrose hin. Die Autoren erforschen den Zusammenhang zwischen Knochendichte und Kniearthrose näher. Dazu untersuchten sie eine Kohorte mit generalisierter hoher Knochendichte und deren gesunde Verwandte hinsichtlich Kniegelenksarthrose.

Die Studienteilnehmer wurden durch die in Großbritannien durchgeführte HBM (high bone mass) Studie rekrutiert. Die Multicenterstudie besteht aus Patienten mit generalisierter hoher Knochendichte unklarer Genese (keine bekannte Erkrankung als Auslöser) und deren nicht betroffene Verwandte. Die Teilnehmer wurden zwischen 2005 und 2010 rekrutiert, die Nachverfolgung fand zwischen 2017 und 2018 statt. Zu Beginn wurden 437 Teilnehmer einge- schlossen, von welchen 274 (63\%) eine hohe Knochendichte aufwiesen. Zum Nachverfolgungszeitpunkt konnte bei 174 Teilnehmern erneut Röntgenaufnahmen durchgeführt werden. Die Knochendichte wurde mithilfe von DXA Aufnahmen bestimmt. Weiterhin wurde durch Röntgenaufnahmen die Klassifizierung von Arthrose nach Kellgren-Lawrence im Kniegelenk vorgenommen. Zusätzlich wurde die minimale Gelenkspalthöhe ermittelt und das Vorhandensein von subchondraler Sklerose geprüft. Alle Teilnehmer füllten einen Fragebogen zu Knieschmerzen, Steifheit, Funktionseinschränkungen, WOMAC-Score und EQ-5D aus. Daten zu Alter, Geschlecht, Bildungsstand und klinische Parameter waren ebenfalls Teil der Erhebung.

Insgesamt konnten 169 Patienten, davon 107 (63\%) mit hoher Knochendichte, in die Auswertung aufgenommen werden. Die durchschnittliche Nachverfolgungsdauer lag bei 8,3 Jahren, das Durchschnittsalter bei 58 Jahren. Die Teilnehmer in der Auswertung waren häufiger weiblich, jünger, körperlich aktiv und weniger oft Raucher oder Diabetiker als Teilnehmer, bei denen keine Daten zum Nachverfolgungszeitpunkt vorlagen. Im Vergleich zu gesunden Teilnehmern, waren Patienten mit hoher Knochendichte eher weiblich, älter und hatten ein höheren BMI. Ein leichter Trend, dass Patienten mit hoher Knochendichte eher zu Kniearthrose neigen als gesunde Teilnehmer, ist erkennbar. Gelenkspaltverschmälerung und Osteophyten traten eher bei Patienten mit hoher Knochendichte auf. Außerdem waren eher Knieschmerzen zu beobachten. Dies ist zu einem großen Teil durch das gehäufte Auftreten von Osteophyten zu erklären.

\section{FAZIT}

Hartley et al. untersuchten, ob bei Patienten mit hoher Knochendichte ein erhöhtes Risiko für Kniegelenksarthrose vorhanden ist. Die Kohortenstudie verglich betroffene Patienten mit ihren gesunden Verwandten. Es konnte keine signifikanten Ergebnisse bezüglich Kniegelenksarthrose festgestellt werden. Patienten mit hoher Knochendichte sind häufiger von Osteophyten und Gelenkspalt- verschmälerung betroffen. Dies führt zu mehr Schmerzen, Steifheit und Funktionseinschränkungen als bei Gesunden.

Stephanie Burgess, Stuttgart 